\title{
Generation of Uniform Laser Beam with Iterative Flexible Dummy Area Method
}

\author{
Yuki Misaki $^{\mathrm{a},{ }^{*}}$, Taiki Nakano ${ }^{\mathrm{a}}$, Toshinori Hora ${ }^{\mathrm{a}}$, Shiyuan Yang ${ }^{\mathrm{a}}$, Fenghui Yao ${ }^{\mathrm{b}}$ \\ ${ }^{a}$ Kyusyu Institute of Technology, 1-1 Sensui-cho Tobata-ku Kitakyuusyuu-shi Fukuoka-ken, 804-8550, Japan \\ ${ }^{\mathrm{b}}$ Tennessee State University, 3500 John Merritt Boulevard Nashville TN,37209-1561,USA \\ *Corresponding Author. misaki@boss.ecs.kyutech.ac.jp
}

\begin{abstract}
Kinoform is a kind of a computer hologram only with a phase modulation, and it hasutilization efficiency of high light in order not to modulate the amplitude of incidence light. kinoform must stabilize amplitude distribution because it does not have amplitude modulation. Iterative dummy area method is an algorithm which reduces the noise of a reproduction image. However, this method is undesirable for error exist in the surrounding dummy domain of an original picture image in utilization efficiency of light.We compare with Iterative dumy area method with flexible dummy area size for the design of kinoform and Iterative dummy area method using the square distribution image. From the result of the reproduction image, there is no obvious noise around an original image.
\end{abstract}

Keywords: kinoform, Iterative dummy area, flexible dummy area.

\section{Introduction}

Kinoform is a kind of a computer hologram only with a phase modulation, and it hasutilization efficiency of high light in order not to modulate the amplitude of incidence light. Kinoform must stabilize amplitude distribution because it does not have amplitude modulation. This brings big error to reproduction images. This error can be reduced by repetition dummy domain method. The problem is that the noise concentrates on the original image by using this repetition dummy domain method. In order to improve this problem, we propose Iterative dumy area method with flexible dummy area size for the design of kinoform. In this paper, we simulate the performance about generation of a uniform laser light using this method, and show the result.

\section{Principle of the kinoform}

Fig.1 shows Fourier transformation type kinoform playback optical system. Two-dimensional intensity distribution should represent in $\mathrm{I}(\mathrm{x}, \mathrm{y})$, sampled data in $\mathrm{I}(\mathrm{m}$, $n$ ), kinoform distribution data calculate by using I (m, n). Kinoform distribution data represented as $\mathrm{W}(\mathrm{k}, \mathrm{l})$. Amplitude distribution of reconstructed image in the sampled point is represented by the following equation.

$g(m, n)=\sum_{k=-\frac{N}{2}}^{\frac{N}{2}-1} \sum_{l=-\frac{N}{2}}^{\frac{N}{2}-1} W(k, l) \exp \left[-\frac{j 2 \pi}{N^{2}}(m k+n l)\right]$

$\mathrm{N}$ is the number of the sampled dispersed points.

Generally, when manufacturing Kinoform, it becomesconstant area distribution using the Kinoform distribution data $\mathrm{W}(\mathrm{k}, \mathrm{l})$. If this is expressed with one dimension, it will become like Fig.2.

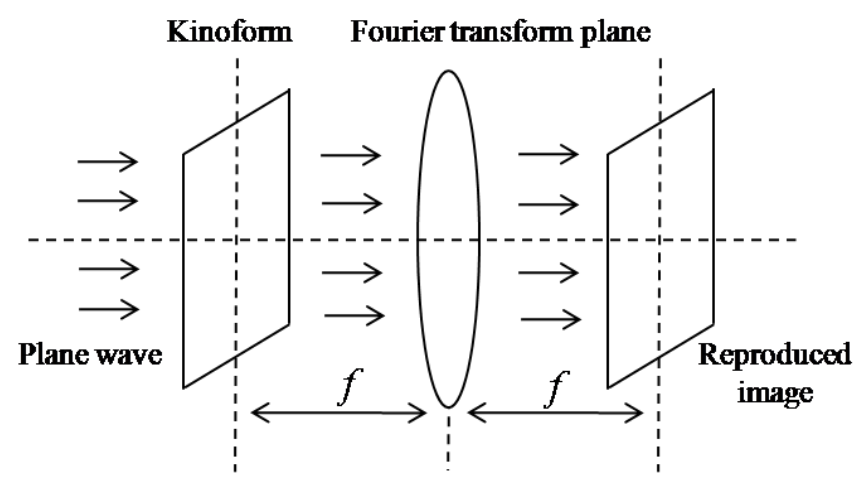

Fig.1. Fourier transformation type 


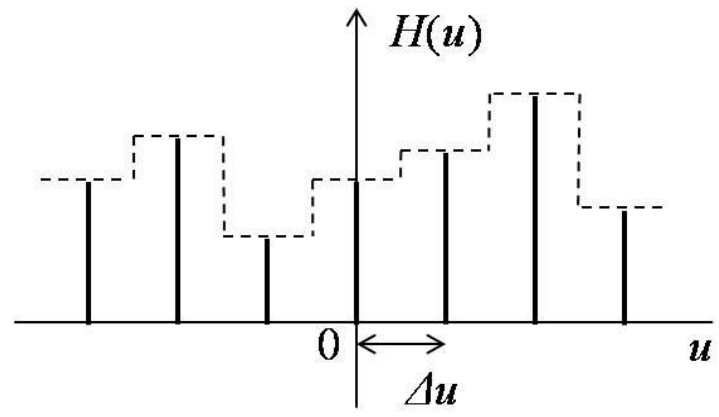

Fig.2. Amplitude distribution of the kinoform

Changediscrete value into continuation value in orderto have constant area. Therefore, collapse a rectangle wave in a discrete value.

$$
\begin{aligned}
& H(u)=W(k) * \operatorname{rect}(u) \\
& k=\operatorname{int}\left(\frac{u+0.5}{\Delta u}\right)
\end{aligned}
$$

$\mathrm{k}$ is the kinoform data number, intis the integer functions. Reproduction image is obtained by Fourier transform $\mathrm{H}(\mathrm{u})$. This become multiply square wave Fourier transform and Fourier transformation of kinoform size distribution data. Because the sinc function is the Fourier transform of the rectangular wave, would put the sinc function in the reconstructed image. Therefore,reproduction image which sampledpoints in the optical playback is computed by followequation.

$$
g^{\prime}(m, n)=g(m, n) * \operatorname{rect}(u)
$$

\section{Iterative dummy area method}

Kinoformmust make amplitude distribution regularity. However, error arises in reproduction image by replacing amplitude distribution uniformly. Iterative dummy area method is an algorithm which reduces this error. The flow of this repetition dummy domain method is shown in Fig.3. Reduction with error is possible by adding the dummy domain of the initial value 0 to the surroundings of an original picture image, use this picture as input picture, using the amplitude of a dummy domainand the flexibility of a phase.

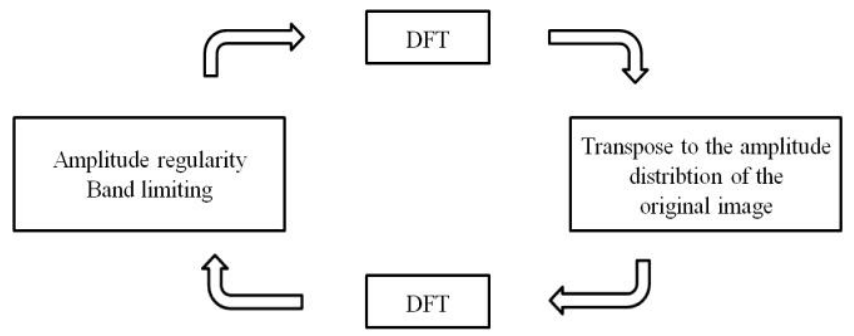

Fig.3. Iterative dummy area method

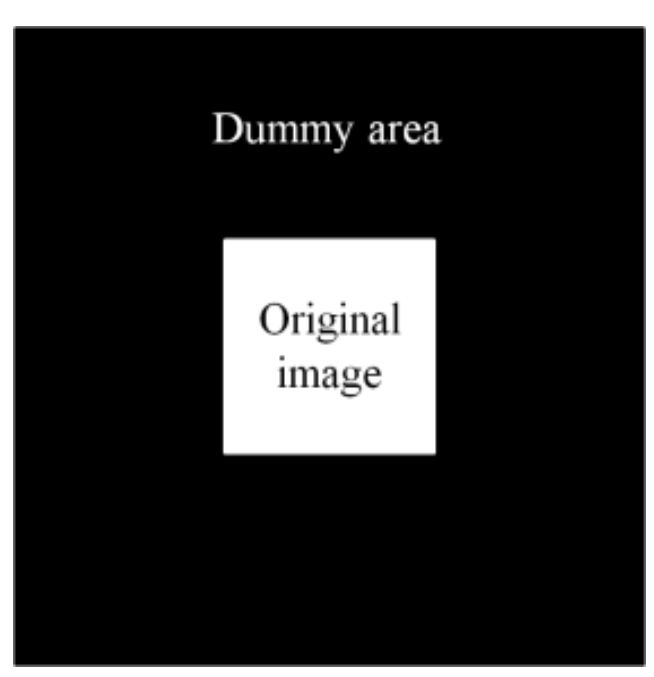

Fig.4. Dummy area

Fig. 4 shows the original image add a blank space. And, using random phaseforthe phase of the original image.

As a restrictioncondition, perform amplitude regularity and band limiting in a hologram side. A reproduction image is obtained by Fourier-transforming the signal after transposing amplitude to a steady value after performing a restriction condition. Moreover, the output obtained by the reproduction image surface is considered as a new input as a restriction condition in the reproduction image surface. It carries out by repeating this.

\section{Iterative dumy area method with flexible dummy area size for the design of kinoform}

In optical reproduction, it is undesirable for error exist in the surrounding dummy domain of an original picture image in utilization efficiency of light. Then, when reproducing a computer hologram, modulatea horizontal direction and a perpendicular direction withsinc function feature which becomes dark. We add variable region to the surroundings of an original picture image.

This variable region including an original picture image set as new original image. This is shown in Fig.5. We performedrepetition dummy domain method to thisimage. Variable region is gradually decreased as a repetition progresses. This variable region changes in a dummy domain at the maximum, in range of an original picture image domain at the minimum. 


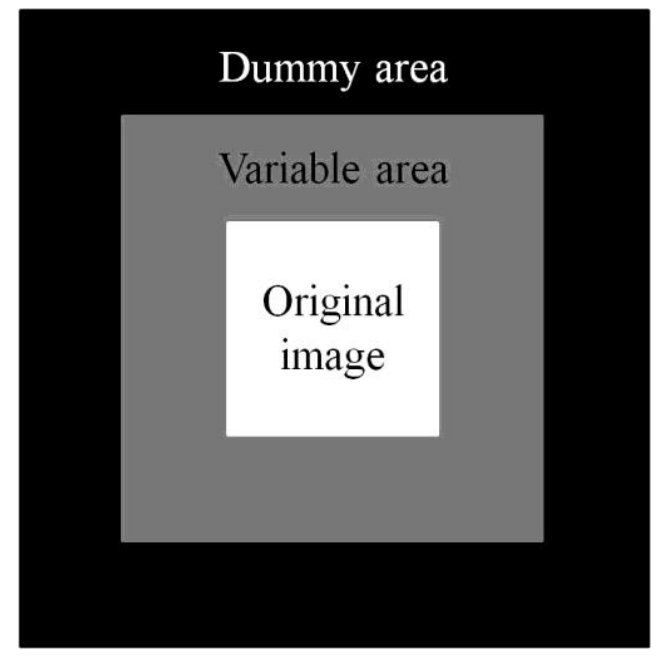

Fig.5. Variable area

\section{Simulation and results}

Show a simulation condition next.

- Images

- Initial phase

- Dummy area

- Band limiting

- It is repeatedly a frequency

We use squareimage of Fig.6 as original picture image. The result of Iterative dummy area method in Fig.7 and The result of Iterative dumy area method with flexible dummy area sizefor the design of kinoform is shown in Fig.8.

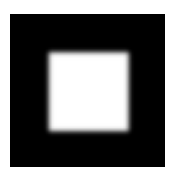

Fig.6. Original image

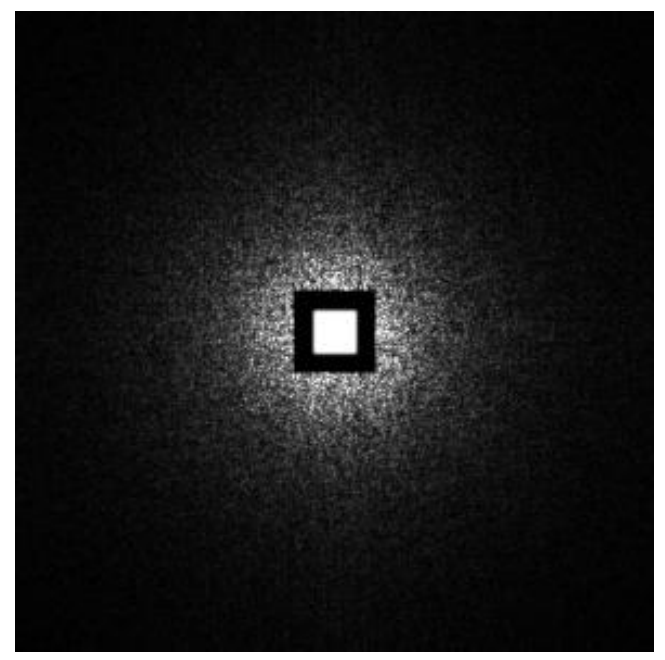

Fig.7. The result of Iterative dummy area method

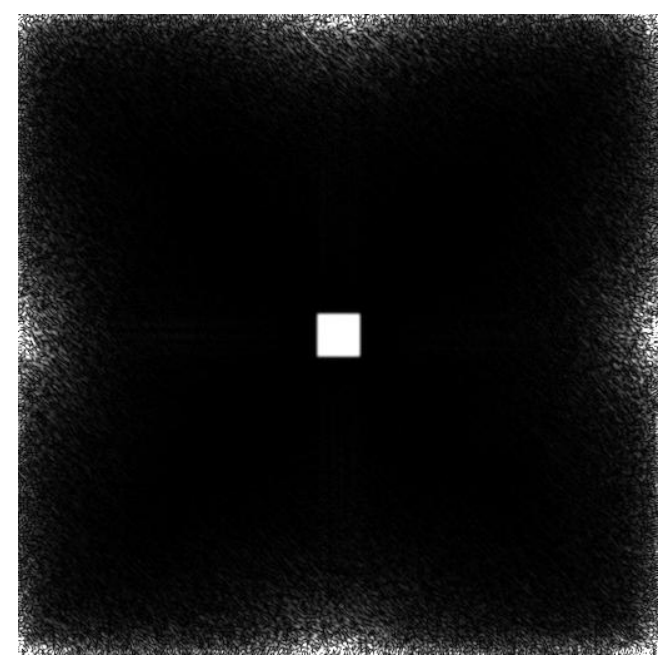

Fig.8. The result of Iterative dumy area method with flexible dummy area size for the design of kinoform

An error produces a lot in the dummy domain around the original image in Fig.7.Compare with Fig.7 and Fig. 8,there is no obviouserror around an original image. In addition, the quality of the reproduction image is same level in comparison with the normal repetition dummy region method.

\section{Conclusion}

Iterative dummy area method is an algorithm which reduces the error of a reproduction image. However, this method is undesirable for error exist in the surrounding dummy domain of an original picture image in utilization efficiency of light.We compare with Iterative dumy area method with flexible dummy area size for the design of kinoform and Iterative dummy area method using the square distribution image. From the result of the reproduction image, there is no obvious noise around an original image. The use efficiency of the light became big, too.

\section{References}

(1) Toshinori Hora, and Shiyuan Yang : "Iterative dummy area method with flexible dummy area size for the design of kinoform”, Proc.of ICIAE2013， 262-272

(2) H. Akahori : "Spectrum leveling by an iterative algorithm with a dummy area for synthesizing the kinoform" Appl. Opt, Vol. 25, No.5, 802-811, 1986

(3) H.Aagedal, M.Schmid, T.Beth, S.Teiwes, and 
F.Wyrowski : "Theory of speckles in diffractive optics and its application to beam shaping", J.Mod.Opt, No.43, 1409-1421, 1996

(4) R.Braer, F.Wyrowski, and O.Bryungdahl : "Diffusers in digital holography", J.Opt.Soc.Am.A, Vol.8, No.3, 572-578, 1991

(5) F.Wyrowski, and O.Bryungdahl : "Speckle free reconstruction in digital holography", J.Opt.Soc.Am.A, Vol.6, No.8, 1171-1170, 1989 